Cahiers « Mondes anciens »

ANCIENS

Histoire et anthropologie des mondes anciens

$6 \mid 2015$

Mères grecques

\title{
Autochtonie et maternité à Athènes (époques archaïque et classique)
}

Autochtony and Maternity in Archaic and Classical Athens

\section{Claudine Leduc}

\section{(2) OpenEdition}

\section{Journals}

Édition électronique

URL : http://journals.openedition.org/mondesanciens/1396

DOI : 10.4000/mondesanciens. 1396

ISSN : 2107-0199

\section{Éditeur}

UMR 8210 Anthropologie et Histoire des Mondes Antiques

Référence électronique

Claudine Leduc, «Autochtonie et maternité à Athènes (époques archaïque et classique) », Cahiers "Mondes anciens » [En ligne], 6 | 2015, mis en ligne le 16 février 2015, consulté le 19 avril 2019. URL : http://journals.openedition.org/mondesanciens/1396; DOI : 10.4000/mondesanciens.1396

Ce document a été généré automatiquement le 19 avril 2019

\section{(c) (i) (9)}

Les Cahiers «Mondes Anciens " sont mis à disposition selon les termes de la licence Creative Commons Attribution - Pas d'Utilisation Commerciale - Pas de Modification 4.0 International. 


\title{
Autochtonie et maternité à Athènes (époques archaïque et classique)
}

\author{
Autochtony and Maternity in Archaic and Classical Athens
}

\author{
Claudine Leduc
}

1 À l'époque démocratique, les palaia, les récits traditionnels des Athéniens, attribuent deux

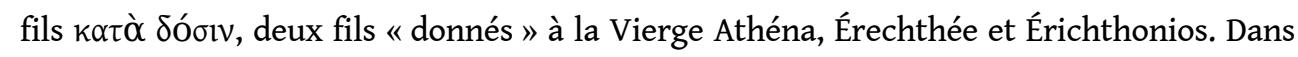

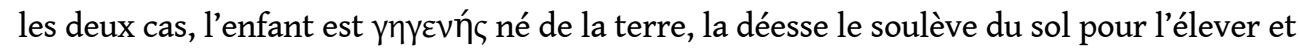

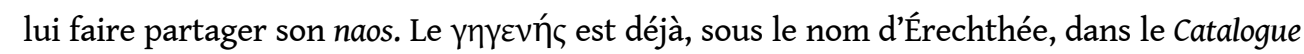
des vaisseaux (Iliade, II, 546-551), le récit fictionnel le plus ancien pour nous, qu'il s'agisse ou non d'une interpolation.

Ensuite ceux d'Athènes, la belle citadelle, dêmos ( $\delta$ ñuov) d'Érechthée, que jadis

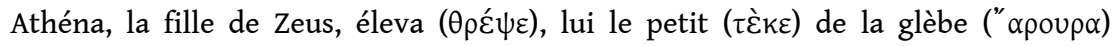
féconde, puis installa à Athènes, dans son gras naos. Aussi est-ce là que les kouroi des Athéniens lui offrent taureaux et agneaux à chaque retour de l'année.

Athènes est le dêmos d'Érechthée ? Comme le précise l'Odyssée (Werlings 2010, p. 49-200) le

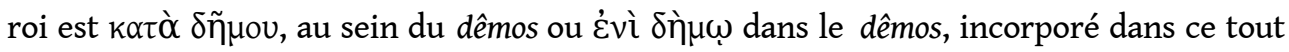
matériel et vivant qui englobe le territoire et la communauté nourrie par lui. La formule

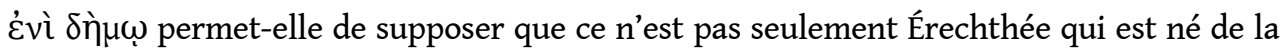
glèbe, mais toute la communauté ? En soulevant Érechthée du sol et en lui faisant partager son naos sur la belle citadelle, Athéna fait de lui son parèdre. Il n'accède pas à

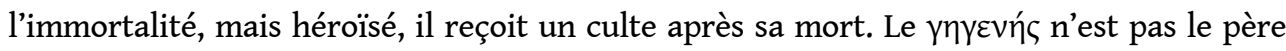
du dêmos, il en fait partie. Il est tout au plus le fondateur de la dynastie royale. Élu par la déesse, il doit vraisemblablement à sa filiation élective le pouvoir qu'il exerce sur le dêmos

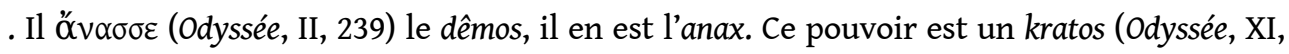
353), une prévalence qui dépend du bon vouloir des dieux et lui vaut d'être écouté comme un dieu. La divine nativité d'Érechthée met en image le fondement théocratique du pouvoir que le roi exerce en tant qu'anax sur le territoire et la communauté territoriale. Athéna est ici, comme elle l'est dans une occurrence bien connue de l'Iliade (VI, 305),

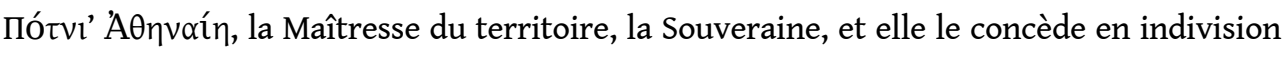
au fils qu'elle s'est choisi. 
3 Transmis donc par la tradition depuis l'époque palatiale, la naissance du үүүعvńs dans les palaia des Athéniens à l'époque démocratique est présentée désormais comme l'acte fondateur de la cité en tant que genos, un groupe de descendance patrilinéaire qui porte le nom de son père fondateur. La fiction « pivote » donc, mais en deux versions.

Les Histoires d'Hérodote attestent par deux fois que, à son époque, la tradition, tout au moins la version qu'il veut bien retenir, nomme le үүүعvท́s, Érechthée.

Dans cette Acropole, il y a un naos d'Érechthée, qu'on dit né de la terre; et dans ce naos, un olivier et une mer qui, à ce que racontent les Athéniens, seraient des témoignages que Poséidon et Athéna auraient laissés de leur contestation au sujet de la possession du pays (Hérodote, VIII, 55).

5 Le genre littéraire auquel appartient le récit d'Hérodote - le genre factuel - veut que le fictionnel (l'intervention d'Athéna) soit occulté et le rituel (le naos) mis au premier plan.

Les habitants de l'Attique [...] étaient des Pélasges et avaient nom Cranaens ; sous le roi Cécrops, ils furent appelés, d'après lui, Cécropides. Lorsque le pouvoir échut à Érechthée, ils avaient changé ce nom en celui d'Athéniens (Hérodote, VIII, 44).

6 Hérodote ne dit pas exactement qu'Érechthée est le fondateur du genos/cité. Mais ses références à Cranaos et à Cécrops, qui donnèrent successivement leurs noms au groupe résidentiel, laissent supposer que, selon lui, il en aurait été ainsi si les habitants n'avaient pas préféré au nom de leur roi - 'E $\rho \varepsilon \chi \theta \varepsilon i ́ \delta \alpha 1$ - celui d'Athéna, les Athéniens. Érechthée siège dans le naos qui contient les "preuves" fournies lors de l'agôn d'Athéna et de Poséidon, l'Érechtheion, sa demeure. Il n'est pas question ici de ses liens rituels avec un autre résident, Poséidon.

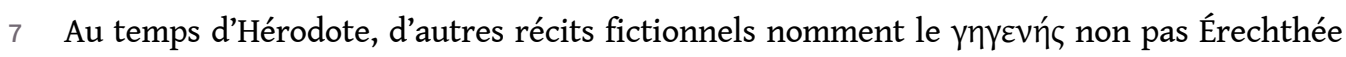
mais Érichthonios. Cette version est celle que, en 418, Euripide, avec Ion (v. 269-272), donne à voir à la cité dans le contexte cérémoniel d'un agôn de tragédie.

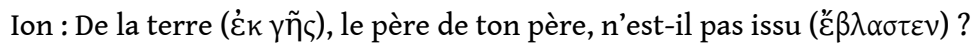

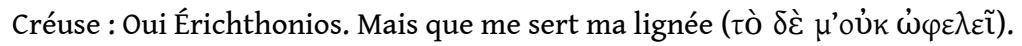

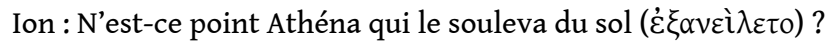

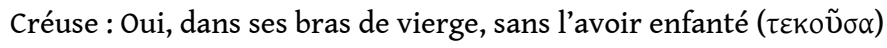

Ion : Et elle le donna, comme on le voit sur les peintures.

Créuse : Oui à garder aux filles de Cécrops, invisible à tous.

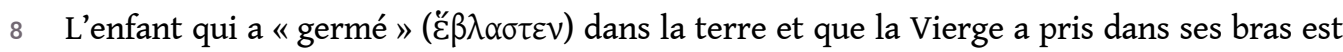
le père du père de Créuse, Érichthonios. Érechthée n'est pas le fondateur du genos, mais son fils. Il est donc rétrogradé à la seconde place dans la généalogie des rois. Ion se fait ensuite préciser (v. 275-281) par Créuse - subtile référence à l'Érechthée d'Euripide joué entre 423 et 422 (Claude Calame 2011, p. 3) -, qu'Érechthée a « sauvé la terre » de son père en immolant ses filles et en mourant foudroyé par Zeus à la demande de Poséidon au cours de sa guerre victorieuse contre Éleusis. Cette mise en scène de la nativité est, au dire du jeune Ion, celle que donnent à voir « les peintures » à tous les Athéniens (Pierre Brulé 1987, p. 68-78), mais surtout entre 475 et 450 ajouterait sans doute Henri Metzger (1976, p. 295-303). Elle est alors, comme dit Jean-Pierre Vernant (1996, p. 239), le miroir où la cité entend se contempler elle-même en contemplant ses origines. Elle demeurera si prégnante que c'est elle que la bibliothèque du Pseudo-Apollodore (III, 188-190) historicisera dans son quadrillage généalogique.

$9 \quad A u V^{e}$ s., Athéna a donc deux fils tous deux nés de la terre. Nicole Loraux, la référence en matière d'autochtonie depuis L'invention d'Athènes (1981), ne s'est pas arrêtée dans Les enfants d'Athéna (1990, p. 41-58) sur cette dualité, car, dit-elle, et à juste titre, « par delà les 
différences, la naissance d'Érichthonios rejoint la mort d'Érechthée, en ce que l'un fonde ce dont l'autre assurera la pérennité ».

Certes! Mais le «bricolage » de la généalogie des rois au bénéfice d'Érichthonios, lui, fait question. Il est possible, me semble-t-il, de l'examiner en prenant deux guides, Nicole Loraux et Claude Calame. À Nicole Loraux revient le principe de la recherche et toutes les découvertes qu'apporta sa mise à l'épreuve. La problématique qu'elle a expérimentée tant de fois, notamment dans Né de la terre (1996) et dans l'ouvrage cher à son cœur, La cité divisée (1997), c'est que les fictions de la tradition religieuse ne doivent pas être mises à l'écart du réel de la cité. Il faut "repolitiser la cité », disait-elle (1986). À Claude Calame revient la méthode d'approche de la tradition religieuse, une méthode qui préconise de mettre en rapport les manifestations symboliques et le contexte historique de leur énonciation et qui n'hésite pas à établir des comparaisons entre le discours fictionnel et le discours factuel. Comme elle n'est plus à rappeler, je me bornerai à préciser deux références. Une citation relevée dans Mythe et histoire. La création symbolique d'une colonie (1996, p. 6) met cette communication en perspective: "Il faudra tenir compte de la production et de la fonction de ces manifestations symboliques dans une situation historique, sociale et idéologique précise.» Un article récent - «Vraisemblance référentielle, nécessité narrative, poétique de la vue» (2012, p. 81-101) - qui démontre que le discours fictionnel, tout autant que le discours factuel, est astreint à la vraisemblance, sera au cœur de mon étude.

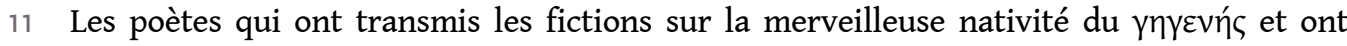
inspiré sa mise en images par les sculpteurs et les imagiers ne sont pas des mythographes qui s'évertuent à mettre de la cohérence dans des versions traditionnelles contradictoires, mais des poiêtai, des créateurs et des guides spirituels qui, dans le cadre rituel d'un agôn de poésie ou de tragédie, et par des procédés qui ne sont pas uniquement narratifs, mettent publiquement en discours un événement que la tradition religieuse considère comme fondateur et qui a une portée idéologique et pragmatique. Ils se doivent de reprendre les données transmises. Mais pour les restituer chargées de sens à la communauté cérémonielle qui les écoutent, ils doivent faire une poiêsis, les réorganiser et les resémantiser en fonction du contexte politique, social et cultuel de leur performance (Leduc 2005). Pour remplir son rôle, la poiêsis doit être une actualisation de la tradition. Mais pour que cette actualisation soit acceptée par l'auditoire, il faut qu'elle soit acceptable, donc qu'elle soit vraisemblable. Ce qui distingue le discours fictionnel du discours factuel, comme lui astreint à la vraisemblance, c'est le langage symbolique de la poésie. Les palaia, à l'époque démocratique, présentent donc deux réactualisations de la

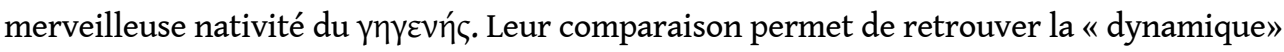
de sa mise en discours.

Quel que soit le récit, la mise en scène de la nativité est centrée sur trois figures étroitement enlacées: Gê en anodos lève les bras pour tendre son tekos à Athéna qui se penche pour le soulever dans ses bras. Faut-il en déduire qu'elle obéit à un seul modèle ? Pour "descendre dans la manifestation discursive ", comme le dit Claude Calame, et montrer qu'elle obéit en fait à des modèles différents, il convient de repérer entre les mises en image ce que j'appellerais « l'intangible » et «le tangible». Sont intangibles : la figure d'Athéna, la figure anthropomorphisée de Gê en anodos, la fabrication d'une filiation élective entre Athéna et l'enfant. Le tangible, c'est la maternité de Gê, dont la conception « pivote », aurait dit Arnold van Gennep, lorsqu'intervient Héphaïstos dans la 
naissance d'Érichthonios. C'est ce «pivotement » qui permet de saisir la « dynamique de la manifestation discursive ».

Dans les deux mises en scène de la nativité, sont intangibles les figures d'Athéna et de Gê. La figure d'Athéna est celle de la Vierge, fondatrice et maîtresse du territoire. En faisant jaillir sur l'Acropole, lors de son agôn avec Poséidon, l'elaia, la fruitière "aux belles olives ", Athéna, grâce à son arbre d'élection, a pris possession du territoire et de la communauté territoriale, du dêmos au sens homérique du terme. Sa domination est réactualisée à chaque retour de l'année par les Panathénées, un rituel réorganisé par Pisistrate (566), mais censé remonter au fondateur de la cité, qu'il soit nommé Érechthée ou Érichthonios. Un tel statut divin dans le système polythéiste d'une cité est unique en pays grec. C'est ce que fait longuement remarquer Pausanias à son lectorat (I, 26, 6). Au cours de sa visite des sanctuaires de la Vieille Grèce, où il est amené à signaler très souvent la présence d'Athéna, il ne verra jamais d'olivier au côté de la Vierge et il ne la qualifiera jamais, même lorsqu'elle a le statut de poliade comme à Élis (VI, 26, 3), Mégare (I, 42, 1-3) ou Tégée (VIII, 45, 4), de fondatrice et de maîtresse du territoire.

La figure d'Athéna est celle de la parthenos. La parthenia, la virginité, a expliqué Giulia Sissa (1987), est en pays grec moins clinique que sociale. C'est le statut de la fille non mariée et l'imaginaire hellénique est plein de parthenioi. Soit! Mais la Vierge est non seulement la fille préférée de Zeus, celle que son père a prise sur ses genoux et qui n'a jamais voulu quitter le giron paternel pour suivre un époux, mais elle est une très chaste jeune fille. Athéna n'est pas au reste la seule déesse à refuser la défloration. Hestia et Artémis se dérobent aussi au pouvoir de Cypris. Mais ce qui fait sa singularité c'est le procédé qu'elle utilise pour y échapper lors de l'Ěpıc, de sa querelle avec Héphaïstos, le dieu des fournaises à la flamme jaillissante, qui brûle de la pénétrer. Elle fuit et se retourne. La fuite de la Parthenos devant son brûlant prédateur, un rôle inhabituel pour Héphaïstos qui, comme elle, a des rapports ambigus avec la reproduction sexuée et la sexualité, était assez connue en pays grec pour avoir été représentée d'après Pausanias (III, 18, 13) sur le trône d'Amyclées. Toutefois au ves., les "peintures " athéniennes s'abstiennent de la mettre en image. La seule représentation en serait, d'après Karl Schefold (1978, fig. 389), l'amphore de Bologne et elle est d'interprétation douteuse, le repérage des attributs identitaires étant aléatoire. La fuite d'Athéna, c'est, dit-on, la scène classique de la fuite de la vierge effarouchée qui, telle Augé, se retourne pour voir si elle arrive à distancer son prédateur, le plus souvent Zeus ou Apollon. La seule différence, c'est que, dans le cas d'Athéna, le retournement est miraculeux puisqu'il lui évite la pénétration. Ce miracle jamais représenté relèverait-il de l'indescriptible? Le refus de la pénétration et le refus de la maternité font partie de ce que Marie Delcourt (1957, p. 11) aurait sans doute appelé "l'être profond" d'Athéna, le substrat intangible de sa configuration.

La figure de Gaia-Gê est aussi intangible que celle d'Athéna. Lorsqu'elle a publié Né de la Terre (1996), Nicole Loraux aurait souhaité ne pas mettre une majuscule à «terre » pour bien souligner qu'il s'agissait de la terre civique et non pas de la Gaia cosmogonique de la Théogonie ou de la "Terre Mère », la figure archétypale des "Généticiens » qui, dans le sillage d'Albrecht Dieterich (1905) en font, comme Pierre Levêque (1985), une mère originelle. Depuis, Stella Georgoudi (2002, p. 120) a repris le dossier en insistant sur le décalage entre le discours pléthorique des Athéniens sur Gaia/Gê et la pauvreté de ses cultes. Gaia/Gê est certes définie par Isocrate (Panégyrique, IV, 24-25) lorsqu'il prétend

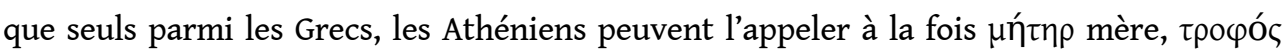


nourrice et $\pi \alpha \tau \rho i ́ c$ terre paternelle, patrie. Mais en fait, dit-elle, pour la désigner, « les Athéniens utilisent de façon parfois interchangeable, et dans le même contexte, d'autres

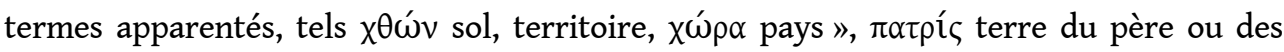
pères, ă $\rho o u p \alpha$ terre labourée, glèbe. Sur la scène de la nativité, la figure anthropomorphisée de Gê en anodos est donc tout cela. Elle est le territoire, mais plus précisément le territoire qui nourrit la communauté. Elle est la terre, allotie autrefois, dont les champs sont la propriété exclusive des citoyens qui se les transmettent de père en fils, les filles étant exclues de la possession du sol. Gaia/Gê est la terre ancestrale que l'on se doit d'aimer à en mourir (Sebillotte Cuchet 2006).

Le face à face entre la maîtresse du territoire et la terre de son territoire est donc la mise en image d'une relation de dépendance. La maîtresse du territoire a la ktêsis sur Gaia/Gê qui ne dispose ni d'elle-même ni de sa progéniture. Elle est dans l'obligation de tendre son tekos à Athéna, qu'il se nomme Érechthée ou Érichthonios.

D'une réactualisation de la nativité à l'autre, la pratique de l'zionoínoıc, de l'adoption entre vifs, permet de penser, Claude Lévi-Strauss (1962, p. 26-33) aurait dit de «bricoler ", le lien de filiation que la Vierge établit avec ses deux fils, Érechthée et Érichthonios.

À Athènes, aussi haut qu'il est possible d'en faire l'histoire, la continuité de la double structuration du groupe civique, la parenté - la transmission des biens - et le politique

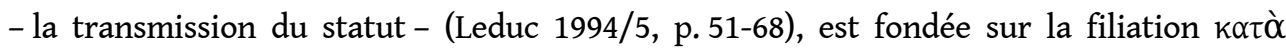
үÉvoc, la filiation biologique. Le père géniteur transmet à ses fils biologiques, à condition

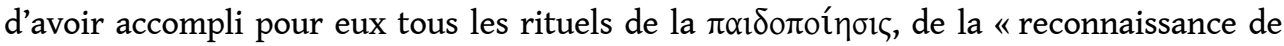
paternité » traduit Jean Rudhart (p. 39-64), ses biens et son statut. Le cas de l'homme ö́aıı, dépourvu de fils, met donc en cause toute la continuité du dispositif. L'adoption (Leduc 1998, p. 175-202) est la procédure qui permet d'assurer sa reconduction de génération en génération. Sa règlementation est attribuée à Solon, mais le législateur a vraisemblablement codifié des pratiques beaucoup plus anciennes. L'homme apais a la

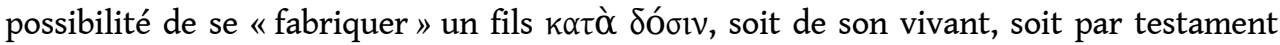

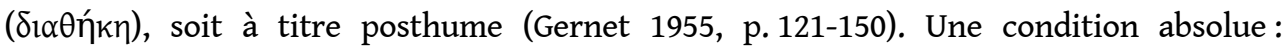
l'adopté doit être le fils légitime du donateur et ce dernier doit être un citoyen en relation de philia avec l'adoptant. Les liens de l'adopté avec son père géniteur sont totalement rompus, mais la relation qu'il entretient avec sa mère biologique et ses parents maternels n'est pas modifiée par la procédure. Que l'adopté soit un enfant ou un adulte, l'adoptant accomplit pour lui les rituels de la paidopoiêsis, sauf ceux de la première année, ( amphidromies, dekatê, meion) qui sont effectués par le géniteur. À l'époque démocratique, en l'introduisant dans sa phratrie, l'adoptant fait de l'adopté son héritier, en l'introduisant dans le dème, il en fait son successeur.

La Parthenos ne pouvant pas avoir de fils kata genos, la pratique de l'eispoiêsis lui permet de procéder à la paidopoiêsis d'un fils kata dosin. En droit attique, un fils kata dosin a les mêmes droits qu'un fils kata genos: son père lui transmet son statut et ses biens. Athéna est la maitresse du territoire, mais il va de soi qu'en tant qu'immortelle elle ne peut le transmettre à son fils adoptif. Le territoire sera donc en indivision entre la déesse, son fils et les descendants de son fils. On objectera qu'Athéna est une femme et qu'une femme ne peut pas se fabriquer un héritier puisque, juridiquement, elle ne peut ni posséder de biens ni les administrer. Elle transmet sa $\pi \rho \circ \ddot{\jmath} \xi$, sa dot en numéraire, de son père à ses fils et son mari en est l'usufruitier (Leduc 1991, p. 259-315). Comme le constate Gabriella Pironti (2013, p. 155-167), lorsqu'il s'agit d'une divinité, la distinction masculin/féminin n'est pas 
forcément un bon critère analytique ! Parce qu'elle est une déesse, Athéna a toute latitude pour se fabriquer un fils kata dosin et pour brouiller toutes les étapes de la paidopoiêsis.

Sur les « peintures » de l'adoption d'Érichthonios, la mise en scène est toujours la même : Gê en anodos offre l'enfant enveloppé dans son lange à Athéna. Ce dernier, toujours un petit mâle à la verge bien apparente, tend avec allégresse les bras à la déesse qui, en simple costume de jeune fille ou en grand apparat de guerrière, se penche et s'apprête à le soulever du sol avec tendresse. Dans les bras de la terre, l'enfant est, comme disent les anthropologues, en humi positio. Cette posture, préalable indispensable à une seconde naissance, est celle de la cérémonie domestique des Amphidromies. Les détails de ce premier rituel de la paidopoiêsis sont assez mal connus (Paradiso 1988, p. 203-218) mais sa portée est claire. Le cinquième jour après sa naissance, le nouveau-né est déposé dans son lange à même le sol devant le foyer de la maison. Son père (?) en le soulevant dans ses bras, lui donne son statut vertical d'homme vivant. En faisant le tour du foyer, il l'intègre dans son oikos. C'est, après la naissance biologique de l'enfant, sa seconde naissance, la première étape de sa fabrication sociale par son père (Bonnard 2003, p. 69-93).

Le rituel des Amphidromies a donc servi de modèle à la mise en scène de la nativité. Mais, si sur les images l'enfant est toujours langé, il est représenté bébé sur le stamnos de Munich $^{1}$, garçonnet sur la coupe du peintre de Kodros ${ }^{2}$, adolescent pubère sur l'hydrie du peintre d'Oinanthé ${ }^{3}$ Athéna, en soulevant l'enfant dans ses bras, concentre donc dans la gestuelle de l'introduction dans l'oikos, tous les rituels de la paidopoiêsis, ceux de la première année comme ceux de l'entrée dans l'âge adulte et le corps civique. L'entrée dans le corps civique - l'obtention de la politeia - est donc donnée à voir comme l'intégration dans l'oikos. Alors que la question de la citoyenneté est une source irréductible de stasis dans la cité, la paisible et lumineuse mise en scène de l'adoption du

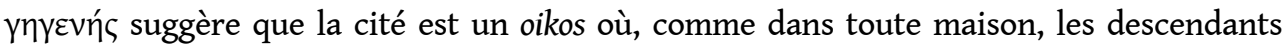
légitimes du père sont des frères qui accèdent à égalité à l'héritage et au statut paternels et qui éprouvent les uns pour les autres de la philia. Cette représentation apaisante des origines serait-elle à mettre en rapport avec une volonté politique d'apaisement des tensions dans le corps civique, celle que Nicole Loraux (1997, p. 202-236) nommait «la politique des frères »?

En dépit des ressemblances de leur mise en images, la naissance d'Érechthée et la naissance d'Érichthonios ne sont pas bâties sur le même modèle. Ce qui « pivote » dans les récits fictionnels, c'est, avec l'introduction d'Héphaïstos, la conception de la maternité de Gê.

Lorsque Gê est la mère d'Érechthée, l'autochtone n'a pas de géniteur. Sa naissance est le résultat d'une parthénogenèse. Gê engendre, enfante et nourrit son tekos et c'est elle qui le donne en adoption à Athéna. La fabrication d'Érechthée n'est donc pas sans analogie avec celle des anciens rois de l'Attique, l'évanescent Actaeos, le célèbre Cécrops et l'énigmatique Cranaos. Tous trois sont, comme lui, engendrés, enfantés et nourris par la terre, mais dans un système d'unifiliation. Ils n'ont ni géniteur, ni père adoptif. Gê procrée seule. Une parthénogenèse ne pouvant mener à son terme la fabrication d'un être humain, les trois fils de la terre sont des hommes-troncs englués jusqu'à la taille dans celle qui les a engendrés. Munis d'une queue de serpent, ils ne peuvent se soulever du sol. Tous trois doivent leur royauté uniquement à leur génitrice. En indivision corporelle avec Gê, ils ne font qu'un avec le pays et ils peuvent lui donner leur nom comme s'ils en étaient eux-mêmes les géniteurs: l'Attique s'est appelée successivement Actê, Cécropeia et Cranae. Aussi, quand ils disparaissent, le pays dans lequel ils sont incorporés ne peut-il 
que disparaitre avec eux. C'est pourquoi ils sont dans l'incapacité de transmettre leur statut à leur descendance. Tous trois sont donc apaides, dépourvus de fils et d'héritiers. Actaios n'a qu'une fille, Aglauros. Cécrops l'épouse, mais lui aussi est apais: son fils Érysichthon, moins célèbre que ses trois filles, meurt très jeune et apais. Cranaos, le successeur de Cécrops, n'a que des filles, dont l'une Atthis donne son nom à l'Attique, et un gendre qui le chasse du pouvoir.

La naissance des rois de l'Attique permet de saisir ce qui fait la singularité de celle d'Érechthée. Gê engendre donc seule l'autochtone, mais elle donne l'enfant à sa divine maîtresse qui, en le soulevant du sol, le sépare physiquement de sa mère - Érechthée n'est pas serpentiforme - et lui donne son statut vertical d'homme. Érechthée a donc une double filiation : il est le fils de Gê et d'Athéna. Pour que la déesse en fasse son fils kata dosin, il faut que la terre l'ait engendré, nourri dans son sein et mis au monde. Fondateur de la lignée royale chez Homère et du genos/cité chez Hérodote, l'autochtone transmet sa double filiation à tous ses descendants qui seront héréditairement procréés par la terre et donnés par elle à Athéna. Ce sont les Érechthéides, un terme qui, d'après Hérodote, aurait immédiatement été remplacé par celui d'Athéniens. Leur nom définit leur statut. Comme leur père, les Érechthéides engendrés par Gê, sont intégrés dans la cité en tant que fils d'Athéna. Pour le dire en langage moins symbolique, ce sont les détenteurs de la terre civique qui sont intégrés dans le corps civique. Leur signe de prédilection est la cigale. C'est-ce que rapporte Thucydide (I, 6, 3) relayé par Aristophane (Les Cavaliers, v. 1321-1334 ; Les Nuées, v. 984-986) qui confirme qu'il s'agit de « vieilleries »:

Il n'y a pas longtemps que les gens âgés des catégories privilégiée portaient encore de longues robes de lin et retenaient la touffe de leurs cheveux en y insérant des cigales d'or.

Aristote (Histoire des Animaux, V, 30, 566b) explique pourquoi la cigale est le signe des Érechthéides. Ils sont fils de la déesse qui a fondé leur territoire en faisant jaillir l'olivier : les cigales, qui adorent les arbres, préfèrent à tous les autres l'arbre d'Athéna à cause de son ombre légère. Ils se disent nés de la terre : les cigales le sont aussi. Au moment du solstice d'été, lorsque l'année a été pluvieuse, les « cigales-mères » (les nymphes) sortent $\mathrm{du}$ sol en grand nombre et déchirent leur enveloppe. Elles déploient leurs ailes, deviennent "noires » et montent dans les oliviers dont la glauque frondaison, en ce moment de l'année, «blanchit » et explose d'éclat de lumière et de stridulations. Aristote explique qu'en dépit des apparences les « cigales-mères » ne sont pas engendrées par la terre. Elles procèdent de la reproduction sexuée. Les femelles, fécondées par le sperme des mâles, pondent des œufs qu'elles déposent dans le sol ou dans des tiges creuses de roseau ou de scille qui rejoignent le sol. Les œufs donnent des larves qui se développent dans la terre, deviennent chenilles puis nymphes. Si les «cigales-mères" semblent procréées par le sol, c'est qu'entre le temps de la ponte des œufs et celui de l'éclosion de la chrysalide, plusieurs années peuvent s'écouler.

Les fils d'Érichthonios n'arborent pas le signe de la cigale. Pourquoi le porterait-il puisque leur père fondateur a une filiation très différente de celle d'Érechthée ? Dans cette fiction, Athéna s'enfuit, se retourne et échappe à l'assaut de son brûlant prédateur, mais l'čpls a une suite : Héphaïstos éjacule sur la cuisse de la Vierge et sa semence tombe sur le sol. Gê la reçoit et tend son petit, nommé Érichthonios, à Athéna qui le soulève du sol.

Comparant la naissance d'Érichthonios à celle de la naissance androgénique et céphalique d'Athéna, Nicole Loraux les interprète toutes deux comme la dénégation symbolique de la maternité de la femme. Zeus accouche d'Athéna à la place de Mêtis, Gê élimine la femme 
de la naissance du fondateur. La femme est invisible dans le «mythe de l'autochtonie » et Athéna n'a que des fils. Cette lecture de la nativité, que certains ont estimée entachée de féminisme, a fait débat. Marcel Detienne (2003) s'est ainsi attaché à réhabiliter la figure de Praxithea dans l'Érechthée d'Euripide comme exemple de la visibilité féminine. La question du genre intervient bien sûr dans le « mythe » de l'autochtonie, mais je ne pense pas qu'elle y soit essentielle. Quatre articles de Nicole Loraux, repris dans Né de la Terre m'ont suggéré une autre hypothèse. Dans le «mythe de l'autochtonie », il est, je crois, moins question de la dénégation symbolique de la femme que de la dénégation symbolique de la terre.

Nicole Loraux remet en cause une phrase générique « qu'historiens et anthropologues de la religion grecque, vont répétant comme une évidence : ce n'est pas la terre qui imite la femme, mais comme le dit Platon, la femme qui imite la terre. Ou encore plus succinctement : les Grecs pensaient que la femme imite la terre ». Viendrait à l'appui de la

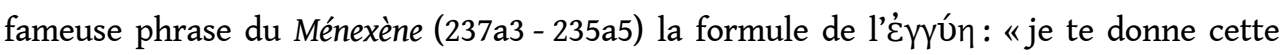
fille à labourer » dit le père de la mariée, en « mettant dans la main » du gendre qu'il s'est choisi, sa fille et sa dot. La reprise, que fait Nicole Loraux du dossier et de son contexte d'énonciation, et chez Platon et dans l'historiographie, autorise me semble-t-il, à renverser le dicton. Dans la fiction de la naissance d'Érichthonios, ce n'est pas « la femme qui imite la terre », c'est « la terre qui imite la femme » ou plus précisément la maternité de la terre est pensée comme celle de la femme. Gê, parce qu'elle est femme, n'engendre pas. Comme il a été souvent fait remarquer, notamment par Lydie Bodiou (2000) et Jean-

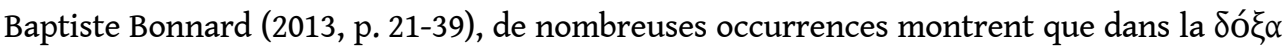
la plus largement partagée, au théâtre comme ailleurs, la femme n'a pas de semence et n'est que le réceptacle de la semence masculine. Combattue par Hippocrate et ses disciples, qui postulaient l'existence de deux semences, cette conception de la reproduction sexuée a été conceptualisée par Aristote et donc promise à un avenir. Dans la Génération des animaux (716a20), il définit ainsi le couple de la reproduction : «Est mâle l'être capable d'engendrer dans un autre, femelle celui qui engendre en soi et de qui naît l'être engendré qui existait dans le géniteur ». Parce qu'elle est femme, Gê est le réceptacle de la semence d'Héphaïstos et son tekos "existait dans son géniteur ». Elle n'est que la matière nourricière de la forme. Héphaïstos est un semeur. Gê, qui reçoit sa semence, en assure la germination. C'est cette image que suggère Euripide dans Ion: Gê fait « germer » - ع̋ $\beta \lambda \alpha \sigma \tau \varepsilon v-$ la semence du dieu. Entre la naissance d'Érechthée et celle d'Érichthonios, Gê passe donc du rôle de génitrice qui enfante seule à celui de mère porteuse dans laquelle « naît l'être engendré ». L'intervention d'Héphaïstos dans la fiction déboute donc Gê de la procréation.

Elle la déboute aussi de l'adoption. En droit athénien, l'adoption est une procédure exclusivement masculine : c'est le géniteur, un citoyen, qui donne un fils à l'adoptant, un citoyen qui fait partie de ses relations. C'est donc Héphaïstos, et non pas Gê, qui donne l'autochtone à Athéna. Les liens nourriciers et affectifs que Gê a noués avec son tekos sont toutefois maintenus. Elle reste celle qui l'a porté, nourri et materné. Sur les images, le dieu, a-t-on fait souvent remarquer, est toujours beau et bienveillant, mais absent, en marge d'une scène qui ne semble pas le concerner. Pourrait-il en être autrement puisqu'il est censé représenter le géniteur qui s'efface devant l'adoptant? La passation de paternité exigeant qu'il y ait entre le géniteur et l'adoptant une relation de philia, le couple paternel que forment Athéna et Héphaïstos doit être un couple de philoi. Le thème de l'Épıc étant incompatible avec leur indispensable philia, les imagiers s'abstiennent de la 
représenter. L'harmonie du "couple paternel», tel est le message que donne à voir l'Héphaïsteion. Bâti au v $\mathrm{v}^{\mathrm{e}} \mathrm{s}$. sur la colline de Kolonos agoraios, ce temple en marbre blanc domine l'agora de Solon. Pausanias précise qu'il est au-dessus du Céramique, ce qui est pour lui une façon de souligner les liens du dieu forgeron avec les artisans de ce quartier. Le temple est destiné à une des figures d'Héphaïstos, celle du géniteur d'Érichthonios. Son naos est la résidence commune des deux pères: la statue d'Héphaïstos " hospite » celle d'Athéna. Le géniteur et l'adoptant sont représentés debout sur le même socle, tandis que, sur la base, Gê tend le fils que l'un a engendré et que l'autre va adopter.

Quelle est l'incidence du pivotement de la maternité de Gê sur la filiation d'Érichthonios et de ses fils, les Athéniens ? Érechthée est engendré par Gê et donné par elle à Athéna : pour être adopté par Athéna, il faut qu'il soit fils de la terre. Érichthonios est engendré par Héphaïstos et donné par lui à Athéna. Gê n'est pour lui qu'une nourrice maternante. Pour qu'il soit adopté par Athéna, il faut qu'il soit le fils de son père Héphaïstos. Il en est de même pour tous les membres de son genos auquel il ne donne pas son nom mais celui de son père adoptif. C'est donc à Athéna que revient la fondation de la cité. Les Athéniens n'en sont pas moins, comme le dit la Pythie au tout début des Euménides (v. 13), les fils d'Héphaïstos. Pour utiliser un langage moins symbolique, pour être fils d'Athéna, pour être intégré par la déesse dans le corps civique, il n'est pas nécessaire d'avoir hérité d'un lot de la terre civique, d'être propriétaire foncier. Il suffit d'être le fils de son père. Pour que les Athéniens soient fils d'Héphaïstos et d'Athéna, il faut qu'Érechthée soit rétrogradé dans la généalogie des rois. Devenant le fils d'Érichthonios ou son petit-fils (lorsque Pandion I est introduit dans la liste), il doit son autochtonie à son père. Ce bricolage généalogique n'a vraisemblablement pas eu d'incidence dans le culte. Érichthonios a une mythopoiêsis qui se limite à l'histoire de sa corbeille de nourrisson confiée aux Cécropides et il est absent du rituel. Comme le remarque Christina Sourvinou Inwood (2011, p. 51-65), Érichthonios est construit avec "le matériel d'Érechthée ». C'est le culte d'Érechthée associé à Poséidon qui est dans le naos de la déesse.

Héphaïstos est donc le géniteur du corps civique alors qu'il a des relations pour le moins problématiques avec la sexualité et la reproduction sexuée. Sa paternité serait-elle à mettre en rapport, comme le suggère Miriam Valdes Guia (2008, p. 89-102), avec le développement, à partir $\mathrm{du} \mathrm{VI}^{\mathrm{e}} \mathrm{s}$., des métiers qu'il patronne au Céramique ? Sa proximité avec Athéna est, je crois, moins " accidentelle ». Dans le système polythéiste athénien, Athéna est en relation très étroite avec Zeus, Poséidon et Héphaïstos, mais c'est avec le Forgeron, l'Épıc mise à part, qu'elle a le plus d'affinités. Tous deux sont des dieux à mêtis, à l'intelligence rusée, des techniciens qui, par leurs inventions, ont apporté la civilisation aux hommes. Tisseuse et ajusteuse incomparables, Athéna a fait jaillir l'elaia, l'olivier femelle et cultivée. Maître de la flamme jaillissante, des fournaises et de toutes les techniques du feu à haute température, Héphaïstos est l'inventeur de la poterie et de la métallurgie. C'est en tant que dieux civilisateurs que tous deux sont honorés ensemble par l'Hymne homérique à Héphaïstos composé vraisemblablement à Athènes dans le courant du $v^{\mathrm{e}} \mathrm{s}$.

Muse harmonieuse, chante l'illustre intelligence d'Héphaïstos qui, avec Athéna aux yeux glauques, apprit les nobles travaux aux hommes de la terre, tandis qu'auparavant ils habitaient des antres dans les montagnes comme des bêtes sauvages.

La Vierge et le Forgeron sont dits de «même nature » dans le Critias (109b-110c). C'est en tant que dieux des technai que le mythe platonicien les installe dans un naos commun sur 
l'Acropole et leur attribue à tous deux la fondation de la cité (109b-110c). Le dieu et la déesse sont honorés ensemble lors de la fête d'automne des Chalkeia appelée aussi Athenaia, ce qui suffit, dit Marie Delcourt (1957, p. 11) à mettre en évidence le rôle de la déesse (Parke 1977, p. 92-93; Simon 1984, p. 51-54). Le 29 de Pyanepsion, les deux arrhéphores et la (les) prêtresse(s) mettent sur le métier le peplos qui sera offert à Athéna dix lunes (une gestation!) plus tard. Le problème, c'est qu'on ne sait pas s'il s'agit d'une fête récente ou ancienne et si le fragment d'une pièce inconnue de Sophocle qui signale la procession du "peuple qui travaille de ses mains" et qui "vans levés» porte des offrandes à «l'Ouvrière aux yeux foudroyants » se rapporte aux Chalkeia (Bérard 1976, p. 101-114). Bref, Héphaïstos et Athéna sont dits très proches. Mais s'ils le sont, c'est à la suite d'une longue histoire.

Si mon analyse des deux nativités n'est pas erronée, les deux mises en scène de la

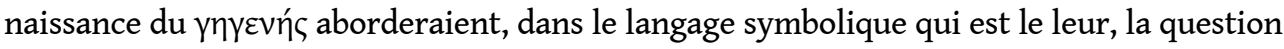
politique fondamentale et récurrente, celle qu'affronte la cité réelle depuis sa réorganisation solonienne en 594/3 jusqu'à la restauration de la démocratie en 403 , celle qu'entendra résoudre, par le biais de la cité idéale, la philosophie au $\mathrm{IV}^{\mathrm{e}} \mathrm{s}$., la question de la politeia, de la citoyenneté (Leduc 1994/5, p. 51-68). Quel garçon, parvenu à l'âge adulte, peut-il devenir fils d'Athéna, être intégré dans le corps civique ? La citoyenneté doit-elle être fondée sur la détention du sol ou sur la naissance? La définition de la politeia/ citoyenneté détermine la politeia/régime politique. Les grandes metabolai des $\mathrm{VI}^{\mathrm{V}} \mathrm{v}^{\mathrm{e}} \mathrm{s}$., comme dit la Constitution des Athéniens (XLI), s'accompagnent d'une redéfinition de la politeia.

Par trois fois - en 594/3 avec Solon, en 508/7 avec Clisthène, en 451/0 avec Périclès - il a été décidé que la politeia du fils serait fondée sur la naissance. Ces mesures sont dites démocratiques puisqu'elles ne tiennent pas compte de la possession du sol et agrandissent le corps civique. Chaque fois, elles sont adoptées dans la stasis et n'ont cessé d'engendrer la stasis. Certes il est très difficile de savoir quelle fut la définition solonienne de la politeia : la Politique et la Constitution des Athéniens ayant fabriqué la « réforme » de Solon en fonction de leur propre idéal politique (Leduc 2012). Mais il convient de remarquer que l'abolition des dettes tant publiques que privées (Constitution des Athéniens, VI, 1), la mesure censée d'après [Aristote] avoir mis un terme à la stasis, est incompatible avec une restriction du corps civique aux détenteurs du sol. Né dans la stasis, le dispositif mis en place par Clisthène établit que sera citoyen le fils légitime d'un père citoyen. Adopté après la période d'affrontement que révèle l'assassinat d'Éphialte (Constitution des Athéniens, XXV), le psephisma dit de Périclès de 451/0 fonde la politeia sur la double filiation: pour être Athénien, il faut être né de deux astoi, d'un père et d'une mère « citoyens » sans que soit précisé que la mère doit avoir été obtenue selon les règles du mariage légitime. Il sera reconduit lors de la restauration de la démocratie en 403. La définition de la politeia sur la naissance ne fut jamais l'objet d'un consensus. Au temps de la démocratie radicale, il y eut toujours des partisans de la réduction du corps civique aux détenteurs du sol comme le prouvent les révolutions oligarchiques de 411 et de 404 . Le critère préconisé alors par les oligarques est toujours la possession du sol, même si certains d'entre eux préfèrent désormais prendre en compte la richesse quelle que soit sa composition.

Le discours traditionnel sur les deux cités, celle d'Érechthée et celle d'Érichthonios, ne met donc pas en image des cités imaginaires. Il dit en langage symbolique ce que dit, sans doute avec plus d'ambiguïté, d'échappatoires et de présupposés idéologiques, le discours 
factuel, en l'occurrence celui de la Politique et de la Constitution des Athéniens, sur les changements apportés dans la définition de la politeia aux $\mathrm{VI}-\mathrm{V}^{\mathrm{e}} \mathrm{s}$. L'autochtonie d'Érechthée, telle que la rapporte Hérodote, est une transposition symbolique de la conception de l'intégration dans la polis qui fut celle de «la politeia primitive », le régime politique mis en place à la disparition de la royauté. Elle est alors une réactualisation de la tradition homérique. Érechthée n'est plus le père de la lignée royale comme il était dans le Catalogue des vaisseaux. La royauté ayant disparu, il devient le père du genos/cité fondé sur la possession du sol. L'autochtonie d'Érichthonios est une réactualisation de l'autochtonie d'Érechthée. Elle exprime symboliquement la définition de la politeia formulée par Clisthène et reformulée par Périclès, celle du régime démocratique. La multiplication des images faisant intervenir Héphaïstos dans la nativité entre 475 et 450 suggère qu'elle est alors devenue prédominante. Au temps de la démocratie radicale, la dernière actualisation de l'autochtonie n'a toutefois pas fait disparaître la précédente dans le discours traditionnel. C'est ce dont témoignent, en 424 et 423, deux occurrences d'Aristophane. La "cité d'Érechthée " et ses cigales relèvent du passé, rétorque le « raisonnement injuste » à l'apologie de l'ancienne éducation dans les Nuées (v. 984-986). Ce sont :

Oui, des vieilleries, qui sentent les Dipolies, avec tout plein de cigales, de Cédidès et de Bouphonies

Mais ces «vieilleries", dans les Cavaliers (v. 1321-1334) restent, pour les adversaires du système démocratique, un idéal de référence. La cuisson de Dêmos dans le chaudron magique doit lui faire retrouver sa jeunesse, sa beauté et... « la cigale dans les cheveux », le symbole de « la cité d'Érechthée », la cité des détenteurs du sol.

Le charcutier : «Je vous ai fait cuire Dêmos et de laid je l'ai rendu beau

Le coryphée : «Quel est son costume ? Quel homme est-il devenu?

Le charcutier : «Le voici qui se présente à vos yeux, la cigale dans les cheveux, dans

tout l'éclat de son antique costume, fleurant bon..."

37 Cette approche diachronique du discours fictionnel des Athéniens sur les deux «maternités blanches» d'Athéna ne porte donc que sur une période historique relativement courte, celle des déstructurations/restructurations du système polis entre la disparition de la royauté et l'installation de la démocratie radicale. Mais, comme l'attestent les données du Catalogue des vaisseaux, il s'agit des réactualisations d'une tradition qui remonte à la période où le roi partageait le naos de la Souveraine sur la «belle citadelle », la période palatiale. En instituant les « fils de la terre » élus par Athéna fondateurs du genos/polis, le récit des deux merveilleuses nativités fait «pivoter » la transmission de la tradition : le «mythe » du fondement théocratique du pouvoir de l' anax devient le «mythe » de la fondation de la cité. En revanche, il reconduit le système divin dont la Vierge en tant que maîtresse du territoire est le noyau organisateur, un système qui prend dans ses mailles, autour d'elle et de l'anax, Poséidon et Héphaïstos. Mais comment tirer les fils cachés de ce réseau hérité de la période palatiale? Si les archives de Cnossos et de Mycènes (?) attestent la présence de la déesse dans le polythéisme mycénien (Rousseau 1968, p. 44-45), il n’y est jamais question de ses figures, de ses fonctions et de ses relations. Je crois, pourtant, qu'il est possible de le tenter.

En se fondant sur la rupture matérielle dont témoigne, sur la plupart des sites, l'effondrement des palais à la fin du $\mathrm{II}^{\mathrm{e}}$ millénaire, nos disciplines établissent une barrière entre la Grèce des palais et la Grèce des cités. Est-elle pleinement justifiée en ce qui concerne la transmission de la culture ? C'est la question que pose Pierre Darcque (1992, 
p. 597-606; 2005, p. 356) à ceux qui s'étonnent que les archives palatiales ne consignent que de la comptabilité et ignorent tout ce qui relèvent de la culture, notamment les représentations religieuses. C'est oublier, dit-il, l'importance, en pays grec, de la transmission orale dont témoignent les poèmes homériques. Il est en effet permis de supposer qu'Athéna n'a pas jailli, lance au point et poussant le cri de guerre de sa terrible voix d'airain, des têtes d'Homère et d'Hésiode. Ses figures, ses exploits et son réseau divin relevaient de la tradition. La culture se transmet de générations en générations, comme le dit Marcel Detienne (1981), par la «bouche et par l'oreille». J'ajouterais à cette belle formule, à la suite d'André Leroi-Gourhan (1964), « et par le geste », car la culture est tout autant transmission des techniques que des «superstructures». Fondée sur l'oralité, sa continuité n'est annihilée ni par l'effondrement des palais ni par la disparition de l'écriture. Elle n'a besoin que de la continuité du peuplement pour se perpétuer. Or la merveilleuse nativité du үпүદvńs relève de la tradition religieuse d'un groupe social enraciné depuis très longtemps sur un territoire et qui a échappé aux grandes destructions de la fin du $\mathrm{II}^{\mathrm{e}}$ millénaire comme aux grandes migrations du $\mathrm{I}^{\mathrm{er}}$ millénaire et où les vestiges de l'organisation palatiale subsistent encore au tout début $\mathrm{du} \mathrm{VI}^{\mathrm{e}} \mathrm{s}$. Cette extraordinaire continuité athénienne entre le $\mathrm{II}^{\mathrm{e}}$ et le $\mathrm{I}^{\mathrm{er}}$ millénaire explique peut-être pourquoi le système polythéiste du palais de Pylos peut offrir quelques ressemblances avec celui du palais de l'Acropole. Ses archives révèlent qu'il existe à Pylos, à la veille de l'effondrement du palais, un système religieux qui, comme à Athènes, met en relation la divine Maîtresse, le wa-na-ka, Poséidon et sinon Héphaïstos, du moins ses forgerons. La lecture des tablettes étant affaire de spécialistes, je me bornerai à quelques notes qui sont en fait de timides questions.

Le nom d'Athéna n'apparaît certes pas dans les archives, mais il y est question d'une mystérieuse po-ti-ni-ja/potnia, d'une Maîtresse sans nom, qui n'est pas dépourvue de ressemblance avec elle! Les tablettes mentionnent le terme po-ti-ni-ja douze fois, tantôt sans épiclèse, tantôt avec des épiclèses de lieux ou de fonctions. Ces potniae avec déterminatif sont-elles des divinités autonomes ou des figures de la Potnia anonyme interpellée selon ses lieux de culte ou ses secteurs de compétence? "Unité ou pluralité » ? S'interroge Claire Boelle (2001, p. 403-409). Cette question relèverait-t-elle de l'impossible? «Desperately seeking potnia » avertissent les éditeurs du colloque Potnia 2001 ? Il est tout de même possible de faire deux remarques. Comme l'avait remarqué Luigia Achillea Stella (1958, p.18-57), les tablettes ne nomment jamais po-ti-ni-ja les divinités féminines du panthéon pylien, ce qui laisse supposer que les potniae à épiclèses sont des figures de la Potnia qu'on ne nomme pas. Parmi les potniae à épiclèses citées, deux d'entre elles, po-ti-ni-ja $i$-qe-ja (PY An 1281) et po-ti-ni-ja a-si-wi-ja (PY Fr 1206) portent des épiclèses qui seront celles d'Athéna au $\mathrm{I}^{\mathrm{er}}$ millénaire. Athéna Hippia est alors une figure très fréquente de la déesse et le nom d'Athéna Asia défie l'imagination des poètes qui le mettent en rapport avec la guerre de Troie. Une troisième épiclèse - $u$-po-jo po-ti-ni-ja- la po-ti-ni-ja du tissage (???), dénote alors une des fonctions d'Athéna Ouvrière.

La Potnia est en relation avec Poséidon. Le système polythéiste de Pylos paraît même structuré autour d'eux. Selon la tablette PY Tn 316, celle qui porte les offrandes exceptionnelles faites dans les sanctuaires à la veille de la destruction du palais, ils seraient les deux grands dieux de Pylos. Au recto, la liste débute avec les offrandes faites à pa-ki-ja-ne, le sanctuaire de la Potnia qui est vraisemblablement proche du palais. Au verso , arrivent en tête les offrandes au po-si-da-i-jo, le sanctuaire de Poséidon situé à sa-ra-pa-da (PY Er 880 et 312), une localisation qui semble périphérique. Les deux sanctuaires sont 
rituellement en relation. Pour la fête re-ke-e-to-tr-ri-jo, la même offrande d'huile parfumée à la sauge est faite à la déesse à la pa-ki-ja-ne (PY Fr 1217) et au dieu à po-si-da-i-jo (PY Fr 343). Apparemment il ne s'agit pas d'une hiérogamie. Poséidon a une parèdre féminine (PY Tn 316). En admettant que la Potnia soit i-qe-ja, Poséidon étant Hippios, leur relation passerait-elle par le cheval et, comme au $\mathrm{I}^{\mathrm{er}}$ millénaire, par le mors du cheval ?

La Potnia et le wa-na-ka, qui est anonyme comme elle, sont en symbiose. Leur relation est vraisemblablement rituelle, mais les archives ne permettent pas de la préciser. La tablette PY Un2 comptabilise des livraisons pour le banquet rituel à l'occasion des initiations, mais le texte ne permet pas de dire si c'est le wa-na-ka qui est intitié, si c'est lui qui procède à des initiations et si la cérémonie a lieu à la pa-ki-ja-ne. En revanche, les tablettes sont prolixes sur les liens économiques et administratifs quasi fusionnels (Rougemont 2009) qui incorporent le sanctuaire de la Potnia dans le palais, notamment en ce qui concerne la détention du sol. La Potnia détient des parcelles de terre distribuées dans plusieurs districts et imbriquées dans les trois systèmes d'occupation du sol (ke-ke-me-na ko-to-na, ki-ki-me-na- ko-to-na et ka-ma). La terre du sanctuaire est allotie. Comme l'a démontré Pierre Carlier (1987, p. 269-273), c'est le palais (Séries PY Eb-Ep) qui procède à la cadastration minutieuse des parcelles et sans doute à la perception de redevances en rapport avec leur superficie. La tablette PY Eq 213 fait état du rapport d'un « inspecteur " et Françoise Rougemont (p. 131) se demande s'il ne s'agirait pas d'un collecteur.

La Potnia est en relation très étroite sinon avec Héphaïstos du moins avec les forgerons (Lejeune 1961, p. 409-434). Les tablettes recensent quelques 400 forgerons (270 sont nommés) dispersés dans 25 localités. Certains de ces forgerons sont po-ti-na-ja-we-jo, de la Potnia. Leur statut est d'autant plus difficile à saisir que les fondements de leur distinction ne sont pas explicités. Selon les tablettes PY Jn 310 et 431, ils n'ont pas d'ateliers particuliers et le fisc ne connait que deux catégories de forgerons, les corvéables et les non-corvéables, ceux qui reçoivent du palais une allocation de bronze et sont astreints à restituer des objets fabriqués (pointes de flèches et de javelots) et les autres. La tablette PY Jn 829, dite " la tablette de la réquisition du bronze des temples » (Rougemont 2009, p. 157-158) éclaire les relations de la Potnia avec la métallurgie et... avec le wa-na-ka. Le bronze réquisitionné dit ka-ko-na-wi-jo, du naos, est prélevé dans 17 localités, ce qui suppose qu'il est entreposé non pas à la pa-ki-ja-ne, mais dans 17 dépôts sacrés. La collecte est faite par des agents du palais et par les dignitaires chargés de l'intendance du sanctuaire de la Potnia. La tablette Jn 829 atteste donc le contrôle absolu de palais sur le bronze de la Potnia, la seule bronzière du panthéon.

Cette incursion dans le palais de Pylos, à propos des «maternités d'Athéna » dans les palaia de la cité démocratique, est-elle intempestive ? Les correspondances entre le réseau divin de la Potnia suggéré par les archives de Pylos et celui d'Athéna révélé par les mise en scène des nativités d'Érechthée et d'Érichthonios, montrent, du moins je l'espère, que lorsque la recherche porte sur la tradition religieuse, il est difficile de s'en tenir à une démarche cloisonnée dans le cadre de la cité. La relation d'Athéna et du Forgeron en est certainement un exemple. Bien avant le développement des arts du feu au vi ${ }^{e} \mathrm{~s}$., elle a été au cœur du système palatial et fut simplement réorganisée et resémantisée dans le contexte historique de la cité. Mais, dira-t-on, qu'en est-il de l'eris de la déesse à l'olivier et du dieu à la flamme jaillissante? La problématique étant différente, elle pourra être l'objet d'une autre communication! 


\section{BIBLIOGRAPHIE}

Bérard Cl. (1976), « Le liknon d'Athéna », AK 19, 2, p. 101-114.

Boelle Cl. (2001), R. Laffineur et R. Hagg éd., « Unité ou pluralité », Potnia 2001. Deities and Religion in the Aegean Bronze Age, Liège, p. 403-409.

Bodiou L. (2000), Histoire du sang des femmes grecques : filles, femmes et mères en Grèce classique, selon les biologistes et les médecins, thèse préparée sous la direction de Pierre Brulé, soutenue à l'Université Rennes 2.

Bonnard J.-B. (2003), « Un aspect positif de la puissance paternelle : la fabrication du citoyen », Mètis N.S. 1, p. 69-93

- (2013), « Corps masculin et corps féminin chez les médecins grecs », CLIO. FGH 37, p. 21-39.

Brulé P. (1987), La fille d'Athènes, Besançon.

Calame Cl. (1996), Mythe et histoire dans l'Antiquité grecque. La création symbolique d'une colonie, Lausanne.

- (2011), « Myth and Performance. Praxithea, Erechtheus, their Daughters. Etiology and Autochtony ", CPh 106, p. 1-48.

- (2012), «Vraisemblance référentielle ; nécessité narrative, poétique de la vue.

L'historiographie classique entre factuel et fictif », Annales HSS, p. 81-101.

Carlier P. (1987), « Palais et sanctuaires dans le monde mycénien », Le système palatial en Grèce et à Rome, Strasbourg, p. 255-290.

Darcque P. (1992), « Recherches et contextes mycéniens : recherches actuelles », dans Olivier J.-P. éd., Mykenaika. Actes du IX colloque international sur les textes mycéniens et égéens. BCH Suppl. 25, Paris, p. 597-606.

- (2005), L'Habitat mycénien. Formes et fonctions de l'espace bâti en Grèce continentale à la fin du $\mathrm{II}^{\mathrm{e}}$ millénaire av. J.-C., Paris.

Delcourt M. (1957), Héphaïstos ou la légende du magicien, Paris.

Detienne M. (1979), « Repenser la mythologie », dans Isard M. et Smith P. éd., La fonction symbolique. Essais d'anthropologie, Paris, p. 71-82.

- (1981), L'Invention de la mythologie, Paris.

- (2003), Comment être autochtone? Du pur Athénien au Français enraciné, Paris.

Dieterich A. (1905 [1925]), Mutter Erde. Ein Versuch über Volksreligion Leipzig.

Georgoudi S. (1994/5), « À la recherche des origines », Mètis IX-X, p. 285-292.

- (2002), « Gaia/Gè. Entre mythe, culte et idéologie », Myth and Symbolic Phenomena in Ancient Greek Culture, Bergen, p. 113-134.

Gérard Rousseau M. (1968), Les mentions religieuses dans les tablettes mycéniennes, Rome.

Gernet L. (1995), « La loi de Solon sur le testament », Droit et société XIII, p. 121-150. 
Leduc Cl. (1991), « Comment la donner en mariage ? La mariée en pays grec du IX au IV ${ }^{\mathrm{e}}$ s. av. J.C., dans Schmitt Pantel P. dir., Histoire des femmes. L'Antiquité, Paris, p. 397-305.

- (1994/5), « Citoyenneté et parenté dans la cité des Athéniens », Mètis IX-X, p. 51-68.

- (2004), « Sur "la nature véritable” du mythe en Grèce ancienne », RHR, p. 475-500.

- (2011), « L'adoption dans la cité des Athéniens », Pallas 85, p. 175-202.

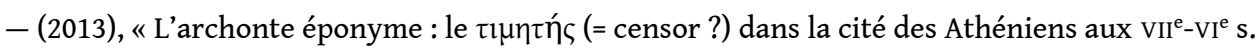
av. J-C. », Mélanges offerts à Jean-Marie Pailler, Pallas 90, p. 201-216.

Lejeune M. (1961), « Les forgerons de Pylos », Historia 10, p. 409-434.

Lévi-Strauss Cl. (1962), La pensée sauvage, Paris.

Leroi- Gourhan A. (1964), Le geste et la parole, tomes 1 et 2, Paris.

Lévêque P. (1985), Bêtes, dieux et hommes. L'imaginaire des premières religions, Paris.

Loraux N. (1986), « Repolitiser la cité », L’Homme 97-98, p. 239-254.

- (1990 [1981]), Les enfants d'Athéna. Idées athéniennes sur la citoyenneté et la division des sexes, Paris.

- (1993 [1981]), L'invention d'Athènes. Histoire de l'oraison funèbre dans la cité classique, Paris.

- (1996), Né de la terre. Mythe et politique à Athènes, Paris

- (1997), La cité divisée. L'oubli dans la mémoire d'Athènes, Paris.

Metzger H. (1976), « Athéna soulevant de terre le nouveau-né : du geste au mythe », Mélanges

P. Collart, Lausanne, p. 295-303.

Godart L., Olivier J-P., Seydel C. et Sourvinou C. (1973), Index généraux du linéaire B, Rome.

Paradiso A. (1988), « L'agrégation du nouveau né au foyer familial : les amphidromies », DHA 14, p. 203-218.

Parke H. W. (1977), Festivals of the Athenians, Londres.

Pironti G. (2013), « Des dieux et des déesses : le genre en question dans la représentation du divin en Grèce ancienne ", Mètis Hors Série 2013, p. 155-167.

Rougemont F. (2009), Contrôle économique et administration à l'époque des palais mycéniens (fin du $\mathrm{II}^{\mathrm{e}}$ millénaire av J.-C.), BEFAR 332, Athènes.

Rudhardt J. (1962), «La reconnaissance de paternité. Sa nature et sa portée », MH 19, fasc. 1, p. 39-64.

Schefold K. (1978), Götter und Helsensagen der Griechse in der Spätarchaischen Kunst, Munich. Sebillotte Cuchet V. (2006), Libérez la patrie. Patriotisme et politique en Grèce ancienne, Paris. Simon E. (1984), Festivals of Attica. An Archaelogical Commentary, Madison.

Sissa G. (1987), Le corps virginal, Paris.

Sourvinou Inwood (2011), dans Parker R. éd., Athenian Myths and Festivals, Oxford.

Stella L. A. (1958), « La religione greca nei testi micenei », Numen 5, p. 18-57.

Valdes Guia M. (2008), El nacimiento de la autoctonia ateniense : cultos, mitos civicos y societad de la Atenas del s. IV AC., Madrid.

Vernant J.-P. (1996), « Formes de croyance et de rationalité en Grèce », Entre mythe et politique, p. 237-252. 
Werlings M. J. (2010), Le dèmos avant la démocratie. Mots, concepts, réalités historiques, Paris.

\section{NOTES}

1. Stamnos à figures rouges du peintre de Munich, Staatliche Antikensammlung, 2413, $A R V^{2} 495$, deuxième quart $\mathrm{du} \mathrm{v}^{\mathrm{e}} \mathrm{s}$.

2. Coupe à figures rouges du peintre de Codros, Berlin, Staatliche Antikensammlung, F 2537, ARV 2 1268 , vers 440 .

3. Hydrie à figures rouges du peintre d'Oinanthé, Londres, British Museum E182, $A R V^{2} 580$, deuxième quart $d u v^{\mathrm{e}} \mathrm{s}$.

\section{RÉSUMÉS}

Hérodote nomme le fils de la terre que la Vierge Athéna a pris dans ses bras pour en faire son fils et l'installer dans son naos, Érechthée. Euripide et les imagiers du v s. le nomment Érichthonios. L'enfant, dans les deux cas, est adopté par la déesse et devient le père fondateur de la polis conçue comme un genos, un groupe de descendance patrilinéaire auquel il donne le nom de l'adoptante. Le discours traditionnel sur la fondation de la cité, pour être accepté par son auditoire et par ceux qui le mettent en images, doit être vraisemblable : il n'est pas plus éloigné des realia que le discours factuel. Repris de générations en générations, il doit être réactualisé en fonction du contexte historique de son énonciation. Dans le langage symbolique qui est le leur, celui de la poésie, les deux versions abordent le grand problème que les Athéniens ont eu à résoudre, de Solon à la restauration de la démocratie en 403, celui de la définition de la politeia, de la citoyenneté. À quelle condition un garçon, parvenu à l'âge adulte doit-il être intégré dans la cité ? La politeia doit-elle être fondée sur la détention du sol ou sur la naissance ? Construit dans un contexte qui est celui de la disparition de la royauté, le récit traditionnel sur la fondation de la cité propose avec «la cité d'Érechthée " un modèle de politeia fondé sur la détention du sol: Érechthée est engendré par la terre. Les données de la tradition sont réorganisées et réactualisées après l'installation de la démocratie. La «cité d'Érichthonios » propose un modèle de politeia fondée sur la naissance : Érichthonios n'est pas engendré par la terre, mais par son géniteur, Héphaïstos, la terre n'est que sa nourrice.

Herodotus names Erechtheus the son of the earth whom the Virgin Athena embraced to mark him out as her son and installs him in her naos. Euripides and the painters of the $\mathrm{v}^{\text {th }} \mathrm{BC}$ name him Erichthonios. In both cases the child is adopted by the goddess and becomes the founder father of the polis, conceived as a genos, a group patrilineal lineage to which is given the name of the adopter: "the Athenians". Both versions are not doublets. The traditional discourse of the foundation of the city must be likely to be accepted by the audience and those who paint it. It is not more remote from the realia than the factual discourse. Rehearsed from generation to generation it must be updated according to the historical context of its statement. In their symbolical language, poetry, both versions deal with the serious problem the Athenians had to solve, from Solon to the restauration of democracy in 403, that is the definition of politeia, the citizenship. What are the conditions to integrate in the city a boy reaching adulthood? Should 
the politeia be founded on the property of earth or by birth? In the historical context of the disappearance of monarchy, the traditional narrative about the foundation of the city proposes with "the city of Erechtheus" a model of politeia based on the property of earth: Erechtheus is engendered by the earth. The facts passed on by tradition are reorganised and reactualised after democracy is institued, "the city of Erichthonios" proposes a model of politeia based on birth : Erichthonios is not engendered by the earth, but by his genitor Hephaïstos, the earth is only his nurse.

INDEX

Mots-clés : Gaia, Gê, Athéna, Héphaïstos, adoption, tradition, réactualisation

\section{AUTEUR}

CLAUDINE LEDUC

Université Toulouse - Jean Jaurès, PLH - EA 4601 